\title{
Licochalcone A in Combination with Salicylic Acid as Fluid Based and Hydroxy-Complex 10\% Cream for the Treatment of Mild Acne: A Multicenter Prospective Trial
}

This article was published in the following Dove Press journal:

Clinical, Cosmetic and Investigational Dermatology

\author{
Federica Dall'Oglio' \\ Gabriella Fabbrocini ${ }^{2}$ \\ Aurora Tedeschi' \\ Marianna Donnarumma $\mathbb{D D}^{2}$ \\ Paolo Chiodini ${ }^{3}$ \\ Giuseppe Micali $\mathbb{D D}^{\prime}$ \\ 'Dermatology Clinic, University of \\ Catania, Catania, Italy; ${ }^{2}$ Section of \\ Dermatology, Department of Clinical \\ Medicine and Surgery, University \\ Federico II of Naples, Naples, Italy; \\ ${ }^{3}$ Medical Statistics Unit, University of \\ Campania "Luigi Vanvitelli”, Naples, Italy
}

Purpose: Topical cosmetic agents if correctly prescribed and used may improve outcomes in acne therapy. The aim of this study was to assess the efficacy and tolerability of a new daily cosmetic regimen in the treatment of mild facial acne.

Patients and methods: A multicenter, prospective, observational, clinical study was conducted on 91 adult patients with mild acne. Subjects were instructed to apply a fluid containing Licochalcone A/Salicylic acid/L-Carnitine in the morning and a cream with Licochalcone A/Hydroxy-Complex $10 \%$ at bedtime for 8 weeks. The efficacy was clinically evaluated by Global Acne Grading System (GAGS) score and by comedones/papules lesions count and by instrumental assessment (Sebutape $^{\mathrm{TM}}$ and Reveal Photo Imager/VISIA-CR ${ }^{\mathrm{TM}}$ imaging) at baseline, at 4 and 8 weeks.

Results: At 4 weeks a statistically significant reduction from baseline of GAGSwas observed. In addition, the mean total count of comedones and papules was significantly reduced by $41 \%$ and $45 \%$, respectively, from baseline along with a significant reduction of mean sebum of $47 \%$. At 8 weeks, a further statistically significant reduction from baseline of GAGS, total count of comedones and papules ( $64 \%$ and $71 \%$, respectively), along with an additional sebum reduction of about $52 \%$ was also recorded.

Conclusion: Our results suggest that the daily regimen based on Licochalcone A with Salicylic acid/L-Carnitine as fluid or with Hydroxy-Complex $10 \%$ as cream represent an interesting cosmetic approach for treating mild acne.

Keywords: mild acne, topical cosmetic, licochalcone A, salicylic acid, hydroxyl-complex

\section{Introduction}

Acne is a common, multifactorial, inflammatory skin disease affecting the pilosebaceous unit resulting from altered keratinization, androgen-induced increased sebum production, inflammation involving both innate and acquired immunity and microbial colonization by Propionibacterium (P.) acnes. ${ }^{1-3}$ Genetic predisposition, anxiety and depression, hyperglycemic diet, sunlight or artificial light, are additional factors known to be involved in the pathogenesis of acne. ${ }^{4}$

Acne is clinically characterized by different clinical aspects and severity, spanning from mild forms, mainly characterized by retentional lesions (RTL) (open and closed comedones) with or without few inflammatory lesions (IFL) (papules/
Correspondence: Giuseppe Micali Dermatology Clinic, University of Catania, A.O.U. Policlinico -Vittorio Emanuele, via Santa Sofia, 78, Catania 95123 , Italy

Tel +39095321705

Fax +390953782425

Email cldermct@gmail.com 
pustules), to moderate or severe forms, with multiple IFL that seriously impact on patient's quality of life. ${ }^{5,6}$

The treatment vary according to disease severity. For mild acne, topical pharmacological treatments are generally recommended and include the use of benzoyl peroxide (BP), retinoid, and antibiotics. ${ }^{5-10}$ The clinical efficacy of these agents is widely demonstrated, and it is well known that their use may cause some adverse effects, such as hypersensitivity reaction, erythema, burning/stinging in case of $\mathrm{BP} /$ retinoids, and limitations for the possible development of resistant bacterial strains for antibiotics. ${ }^{5-10}$

Recent evidence suggests that some cosmetic agents if correctly prescribed may improve the therapeutic outcome with the advantage of minimizing the side effects and enhance the efficacy of prescription drugs. ${ }^{11}$

The aim of this study was to assess the efficacy and tolerability of a new cosmetic regimen consisting in the daily use of an anti-inflammatory/corneolytic/sebum controlling fluid in the morning and of an anti-inflammatory /corneolytic cream at bedtime in the treatments of mild facial acne by clinical and instrumental evaluations for 8 weeks duration study.

\section{Materials and Methods Study Design}

This was a multicenter, prospective, observational clinical study.

\section{Setting and Study Period}

From October 2017 to May 2018 ninety-one adult patients $(71 \mathrm{~F} / 20 \mathrm{M})$ with mild acne were enrolled at the Dermatology Department of the University of Catania (Italy) and of University "Federico II" of Naples (Italy). Study duration was 8 weeks. The study was performed in accordance with the ethical principles originating from the Declaration of Helsinki 1996 and Good Clinical Practices. The protocol was approved by an internal review board. A written informed consent was obtained from each patient before study procedures were started, which included consent to use their images.

\section{Inclusion/Exclusion Criteria}

Inclusion criteria were: adult patients with mild facial acne (open and closed comedones and up to 10 papules), a wash out period of at least 2 weeks for any topical acne treatments, and 1 month for oral antibiotics and 2 months for hormonal therapy and/or oral isotretinoin. Exclusion criteria were: presence of pustules or nodules lesions, trunk localization, severe underlying diseases, concurrent exposure to sunlight and/or artificial ultraviolet sources, pregnancy and breastfeeding. No other topical products or drugs were allowed, except for a mild cleanser, make-up and oil-free SPF 50+ sunscreens.

\section{Methodology}

Patients were instructed to apply a fluid containing Licochalcone A combined with Salicylic acid, and L-Carnitine in the morning and a cream based on Licochalcone A and Hydroxy-complex $10 \%$ at bedtime for 8 weeks. A mild, perfume-free cleaning gel principally based on Ampho-Tenside 6\% was suggested for daily care. In order to reduce potential evaluator bias, all subjects were assessed by an investigator not directly involved in the study at baseline (T0), and at 4 weeks (T1), and 8 weeks (T2).

\section{Clinical and Instrumental Evaluation Criteria}

Clinical and instrumental evaluation were carried out at baseline, and at 4 and 8 weeks.

Acne severity was rated by clinical evaluation using: 1) the Global Acne Grading System (GAGS) score consisting in a subjective assessment of acne severity proposed by Doshi et al in 1997 that divides the face into six areas, forehead, nose, chin, right cheek, left cheek, chest and upper back and assigns a factor (from 1 to 3 ) to each area $(2=$ forehead; $1=$ nose; $1=$ chin; $2=$ right cheek; $2=$ left cheek; $3=$ chest and upper back): the local score is calculated using the formula factor $\mathrm{x}$ grade (depending on the most severe lesion type; $0=$ no lesions; $1=$ comedones; 2=papules; 3=pustules; 4=nodules); the global score is the sum of all global scores: a score of 1-18 is considered mild; $19-30$ moderate; $31-38$ severe and $>39$ very severe and by 2) lesions count, method based on count of the total number of retentional and/or inflammatory lesions. ${ }^{12,13}$

Instrumental assessment included: 1) measurement of sebum by Sebutape ${ }^{\mathrm{TM}}$ strips (CuDerm Corp., Dallas, TX, USA) placed on the patient's forehead, nose, cheeks, and chin and subsequently checked against a black background of a score card; sebum spots are scored on a scale ranging from 1 to 5 , where 1 indicates a dry skin without sebum, and 5 identifies a very oily skin, and 2) facial imaging performed by high-tech facial photography characterized 
by 15 mega pixel resolution and flash cross-polarized Light by Reveal Photo Imager (Canfield Scientific Inc., Parsippany, NJ, USA) at Naples site and by VISIA-CR ${ }^{\text {TM }}$ imaging system (Canfield Scientific Inc., Fairfield, NJ, USA) at Catania site. ${ }^{14-16}$

Additionally, evaluation of product tolerability by a self-administered questionnaire based on 5 parameters (erythema, scaling, dryness, stinging/burning and itch) from 0 to 3 ( 0 =none; $1=$ mild; $2=$ moderate; $3=$ =severe $)$ was carried out.

\section{Study Endpoints}

Primary endpoint was the clinical efficacy at week 4 and 8 evaluated by reduction of GAGS score and lesion count; secondary endpoint was the evaluation of tolerability and cosmetic acceptability at the end of the study.

\section{Statistical Analyses}

The characteristics of the patients were reported as absolute number and percentage for the categorical variables and as mean and standard deviation (SD) for the continuous variables. The analysis of longitudinal data was performed using a mixed effect regression model, which considered the correlation between measurements carried out over time in the same subject. In the mixed effect regression models, time, age, gender and center were considered as covariates. Interaction between center and time was also tested. For time covariate Dunnett post-test was used for multiple comparisons vs baseline. A twotailed $\mathrm{P}$ value $<0.05$ was considered significant. All statistical analyses were performed using SAS version 9.4 (SAS, Inc., Cary, NC).

\section{Results}

Ninety-one subjects (71F/20M; age range: $18-30$ years; mean age $21.5 \pm 3.8$ years; mean GAGS: $11.6 \pm 2.4$ ) with mild acne were enrolled. Eighty-eight (70F/18M) completed the study whereas three subjects were lost to follow-up for personal reasons.

At 4 weeks a statistically significant reduction from baseline of GAGS (mean from 11.6 \pm 2.4 to $10.3 \pm 3.0$; $\mathrm{p}<0.001$ ) was observed. In addition, the mean total count of RTL and IFL was significantly reduced of $41 \%$ and $45 \%$, respectively, from baseline (comedones: mean from $42.3 \pm 14.3$ to $24.8 \pm 12.7 ; \mathrm{p}<0.001$; papules: mean from 5.5 \pm 5.0 to $3.0 \pm 3.8 ; \mathrm{p}<0.001)$ along with a significant reduction of mean sebum of about $47 \%$ (mean from $2.13 \pm 0.67$ to $1.13 \pm 0.38 ; \mathrm{p}<0.001$ ) (Table 1 , Figure 1).

At 8 weeks a further statistically significant reduction from baseline of GAGS $(8.2 \pm 2.9 ; \mathrm{p}<0.001)$ was also recorded; similarly the mean total count of RTL and IFL was significantly reduced of $64 \%$ and $71 \%$, respectively, from baseline (comedones: mean from $42.3 \pm 14.3$ to 15.0 \pm 9.6 ; papules: mean from $5.5 \pm 5.0$ to $1.6 \pm 3.0 ; \mathrm{p}<0.001)$, along with an additional mean sebum reduction of about $52 \%(\mathrm{p}<0.001)$ (Table 1, Figure 2). For all considered

Table I Results from Clinical and Instrumental Evaluation of GAG Score, Total Lesion Count of Comedones/Papules from Baseline to 8 Weeks

\begin{tabular}{|c|c|c|c|c|c|c|c|}
\hline \multirow[t]{2}{*}{ Evaluations (Mean Value) } & \multicolumn{2}{|c|}{ Baseline } & \multicolumn{2}{|c|}{4 Weeks } & \multicolumn{2}{|c|}{8 Weeks } & \multirow[t]{2}{*}{ P Value } \\
\hline & Mean & DS & Mean & DS & Mean & DS & \\
\hline \multicolumn{8}{|l|}{ GAGS } \\
\hline Catania & 11.2 & 2.2 & 9.6 & 3.4 & 7.7 & 3.3 & $<0.001$ \\
\hline Naples & 12.0 & 2.6 & 10.9 & 2.6 & 8.7 & 2.4 & $<0.001$ \\
\hline Overall & 11.6 & 2.4 & 10.3 & 3.0 & 8.2 & 2.9 & $<0.001$ \\
\hline \multicolumn{8}{|l|}{ Total Count Comedones } \\
\hline Catania & 39.3 & 11.9 & 20.8 & 13.6 & 12.6 & 10.9 & $<0.001$ \\
\hline Naples & 45.1 & 16.0 & 28.7 & 10.5 & 17.2 & 7.6 & $<0.001$ \\
\hline Overall & 42.3 & 14.3 & 24.8 & 12.7 & 15.0 & 9.6 & $<0.001$ \\
\hline \multicolumn{8}{|l|}{ Total Lesions Papules } \\
\hline Catania & 4.2 & 3.3 & 2.3 & 2.4 & 1.3 & 2.0 & $<0.001$ \\
\hline Naples & 6.7 & 6.0 & 3.7 & 4.8 & 1.8 & 3.7 & $<0.001$ \\
\hline Overall & 5.5 & 5.0 & 3.0 & 3.8 & 1.6 & 3.0 & $<0.001$ \\
\hline \multicolumn{8}{|l|}{ Sebutape } \\
\hline Naples & 2.13 & 0.67 & 1.13 & 0.38 & 1.03 & 0.37 & $<0.001$ \\
\hline
\end{tabular}



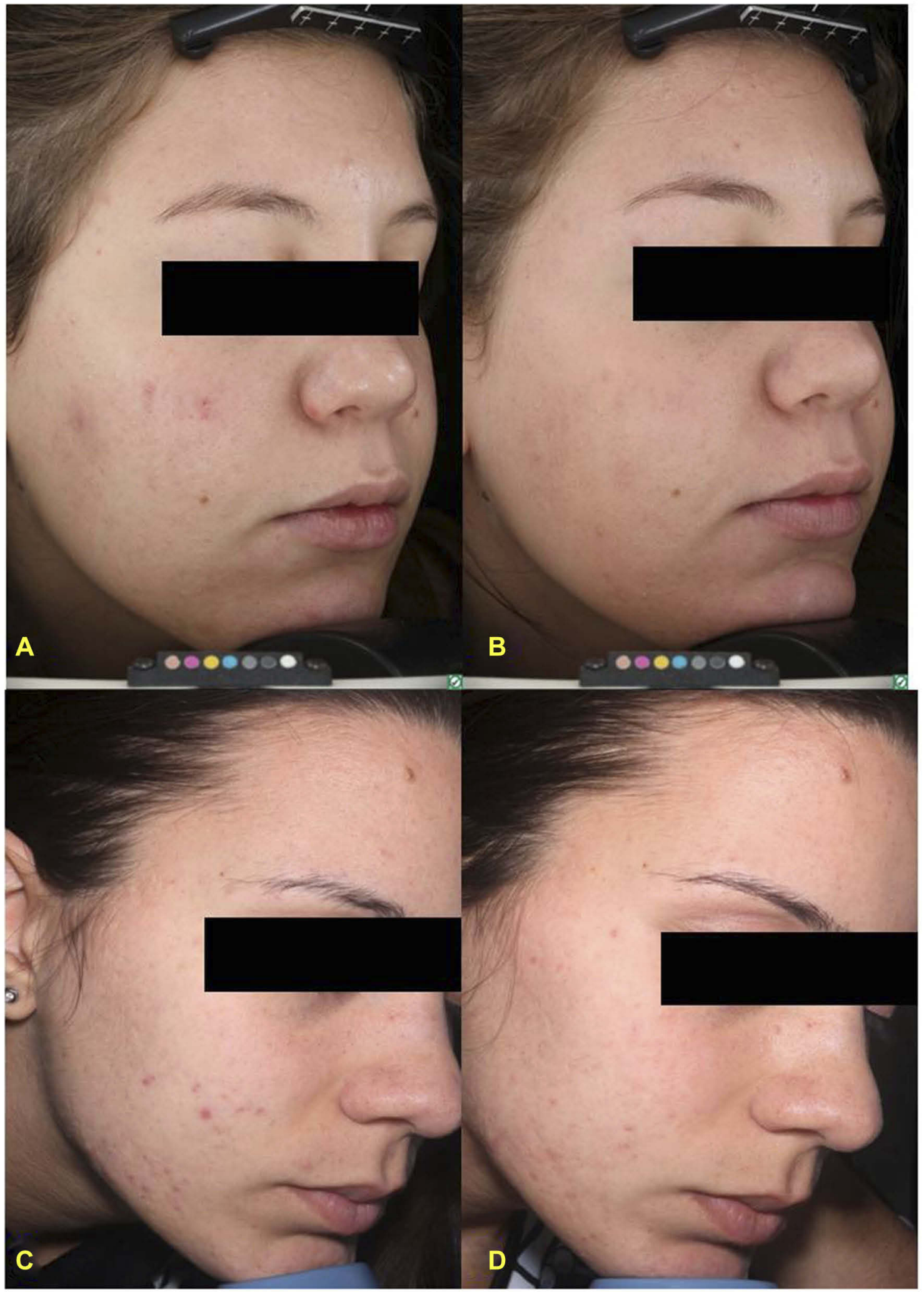

Figure I Advanced digital photography of two patients affected by mild acne at baseline $(\mathbf{A}, \mathbf{C})$ and after 4 weeks of treatment $(\mathbf{B}, \mathbf{D})$.

outcomes, Dunnett multiple comparison post-tests versus baseline showed a significant reduction $(\mathrm{p}<0.001)$ of 4-weeks and 8-weeks values. The two centers showed similar results in the reduction of GAGS (interaction center*time, $\mathrm{p}=0.655$ ) and total count of comedones (interaction center*time, $\mathrm{p}=0.192$ ), while for total count of 


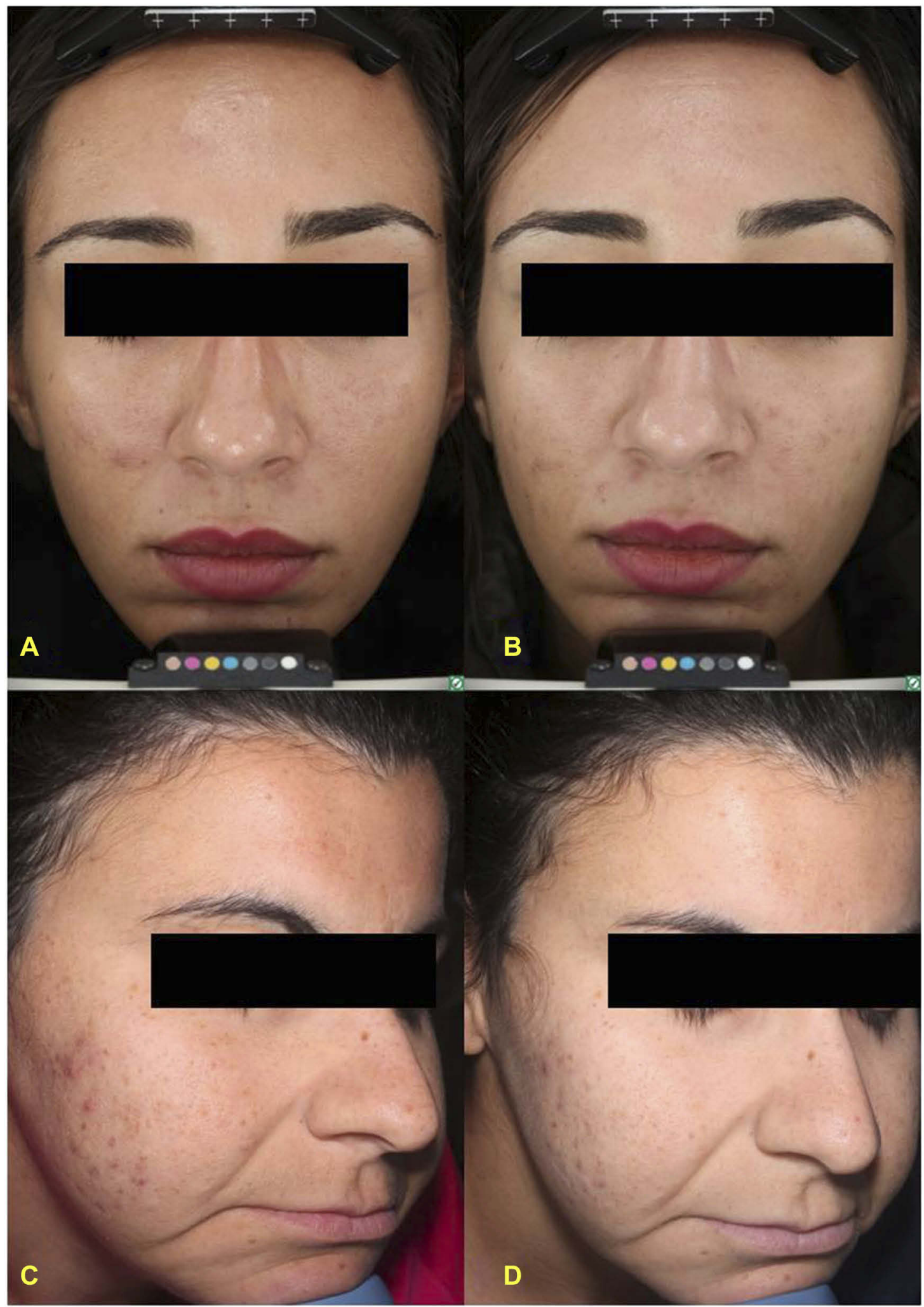

Figure 2 Advanced digital photography of two patients affected by mild acne at baseline $(\mathbf{A}, \mathbf{C})$ and after 8 weeks of treatment $(\mathbf{B}, \mathbf{D})$.

papules a significant interaction center*time was found $(p=0,007)$ showing a greater reduction in the Naples center in comparison to Catania center (Table 1).
Local side effects were documented in 3 cases only (severe erythema: 1 case; severe hitch: 2 cases) and product tolerability was rated as excellent in $90 \%$ of patients. 


\section{Discussion}

Cosmetic treatment for acne, if correctly prescribed and used, may improve outcomes in acne therapy. ${ }^{11}$ The topical cosmetic approach for acne treatment includes: hygiene and cleansing, ideally with a non-comedogenic, non acnegenic, non-irritating and non-allergenic cleanser; sebum controlling agents, generally based on the so-called "mattifyng" ingredients able to absorb sebum from skin surface, in case of hyperseborrea; corneolytics agents, particularly indicated for comedonic acne as able to induce a comedolytic effect, making comedones more superficial and at the same time may also facilitate skin absorption of other products; antimicrobial agents that have been proven to effectively reduce P.acnes growth both in vitro and in vivo clinical study; anti-inflammatory agents indicate to control the pivotal role of inflammatory events in the development of acne lesions; moisturizers product specifically designed for acne patients that improve adherence to therapy by alleviating the discomfort and feeling of dryness following topical and systemic retinoids or topical BP. ${ }^{11}$ The use of specific photoprotective agents and shaving products should also be encouraged. Finally, tailored camouflage make-up technique using non-comedogenic products may minimize the esthetic problem associated with acne and improve patients' self-esteem and adherence to treatment. ${ }^{11}$

The results of our study suggest that the tested products are effective in the treatment of mild acne achieving a good resolution in cure rates due to a synergic action of its agents concurrently able to control different factors involved in the pathogenesis of acne.

In detail, licochalcone $\mathrm{A}$ is a natural flavonoid substance isolated from Glycyrrhiza glabra and Glycyrrhizainflata (licorice root) with anti-inflammatory and anti-microbial effects, as confirmed by inhibition in vitro and in vivo of pro-inflammatory cytokines (PGE2, LTB4, IL-6 and TNF$\alpha),{ }^{14-15}$ as well as anti ROS-induced cell damage action by activating of the expression of antioxidant transcription factor (Nf-E2-related factor 2/Nrf2) and of detoxifying enzymes (Heme Oxygenase 1/HO-1, Glutamate-Cysteine Ligase Modifier subunit/GCLM). ${ }^{17-19}$ Hydroxy Complex is a combination of three exfoliating agents (Glycolic, Salicylic and Polyhydroxy Acids) able to cause intercorneocyte cell detachment so as to induce a comedolytic effect. $^{20-22}$ Finally, L-Carnitine is a sebum controlling agent by $\beta$-oxidation of intracellular lipid content as demonstrated in vitro and in vivo clinical study. ${ }^{23}$

\section{Conclusions}

Our results suggest that the daily regimen-based Licochalcone $\mathrm{A}$ in combination with Salicylic acid/L-carnitine as fluid or with Hydroxy-Complex 10\% as cream represent an interesting approach for treating mild acne that undoubtedly deserves attention. It may represent a significant adjunct to the therapeutic armamentarium for the management of acne patients without any significant side effects. We are aware that our study has some restrictions being a non-controlled trial but it was a multicenter study with a considerable number of patients enrolled. Further studies on larger series of adolescent acne patients are necessary to confirm our results.

\section{Disclosure}

The study was supported financially by Beiersdorf SpA (Milano, Italy), who manufacture the product. Paolo Chiodini received an honorarium for a consultancy from Beiersdorf SpA (Milano, Italy). The other authors declare no other conflicts of interest in this work.

\section{References}

1. Gollnick HP. From new findings in acne pathogenesis to new approaches in treatment. J Eur Acad Dermatol Venereol. 2015;29 (Suppl 5):1-7.

2. Kircik LH. Advances in the understanding of the pathogenesis of inflammatory acne. J Drugs Dermatol. 2016;15(1 Suppl 1):s7-s10.

3. Picardo M, Eichenfield LF, Tan J. Acne and Rosacea. Dermatol Ther (Heidelb). 2017;7(Suppl 1):43-52. doi:10.1007/s13555-016-0168-8

4. George RM, Sridharan R. Factors aggravating or precipitating acne in Indian adults: a hospital-based study of 110 cases. Indian J Dermatol. 2018;63(4):328-331. doi:10.4103/ijd.IJD_565_17

5. Zaenglein AL, Pathy AL, Schlosser BJ, et al. Guidelines of care for the management of acne vulgaris. J Am Acad Dermatol. 2016;74 (5):945-973. doi:10.1016/j.jaad.2015.12.037

6. Bahali AG, Bahali K, BiyikOzkaya D, et al. The associations between peer victimization, psychological symptoms and quality of life in adolescents with acne vulgaris. J Eur Acad Dermatol Venereol. 2016;30(12):e184-e186. doi:10.1111/jdv.13495

7. Gollnick H, Cunliffe W, Berson D, et al. Management of acne: a report from a Global Alliance to Improve Outcomes in Acne. J Am Acad Dermatol. 2003;49(Suppl 1):S1-S37. doi:10.1067/ mjd.2003.618

8. Strauss JS, Krowchuk DP, Leyden JJ, et al; American Academy of Dermatology/American Academy of Dermatology Association. Guidelines of care for acne vulgaris management. $\mathrm{J} \mathrm{Am} \mathrm{Acad}$ Dermatol. 2007;56(4):651-663. doi:10.1016/j.jaad.2006.08.048

9. Thiboutot D, Gollnick H, Bettoli V, et al; Global Alliance to Improve Outcomes in Acne. New insights into the management of acne: an update from the Global Alliance to Improve Outcomes in Acne group. $J$ Am Acad Dermatol. 2009;60(Suppl 5):S1-S50. doi:10.1016/j.jaad.2009.01.019

10. Nast A, Dréno B, Bettoli V, et al.; European Dermatology Forum. European evidence-based (S3) guidelines for the treatment of acne. J Eur Acad Dermatol Venereol. 2012;26(Suppl 1):1-29.

11. Dall'Oglio F, Tedeschi A, Fabbrocini G, et al. Cosmetics for acne: indications and recommendations for an evidence-based approach. G Ital Dermatol Venereol. 2015;150(1):1-11. 
12. Doshi A, Zaheer A, Stiller MJ. A comparison of current acne grading systems and proposal of a novel system. Int $J$ Dermatol. 1997;36:416-418. doi:10.1046/j.1365-4362.1997.00099.x

13. Becker M, Wild T, Zouboulis CC. Objective assessment of acne. Clin Dermatol. 2017;35(2):147-155. doi:10.1016/j.clindermatol.2016.10.006

14. Fabbrocini G, Capasso C, Donnarumma M, et al. A peel-off facial mask comprising myoinositol and trehalose-loaded liposomes improves adult female acne by reducing local hyperandrogenism and activating autophagy. J Cosmet Dermatol. 2017;16(4):480-484. doi:10.1111/jocd.2017.16.issue-4

15. Goldsberry A, Hanke CW, Hanke KE. VISIA system: a possible tool in the cosmetic practice. J Drugs Dermatol. 2014;13(11):1312-1314.

16. Micali G, Dall'Oglio F, Tedeschi A, Lacarrubba F. Erythema-directed digital photography for the enhanced evaluation of topical treatments for acne vulgaris. Skin Res Technol. 2018;24(3):440-444. doi:10.1111/ srt.2018.24.issue-3

17. Kolbe L, Immeyer J, Batzer J, et al. Anti-inflammatory efficacy of Licochalcone A: correlation of clinical potency and in vitro effects. Arch Dermatol Res. 2006;298:23-30. doi:10.1007/s00403006-0654-4
18. Angelova-Fischer I, Rippke F, Fischer TW, et al. D.A double-blind, randomized, vehicle-controlled efficacy assessment study of a skin care formulation for improvement of mild to moderately severe acne. $J$ Eur Acad Dermatol Venereol. 2013;27(Suppl 2):6-11. doi:10.1111/ jdv.2013.27.issue-s2

19. Kühnl J, Roggenkamp D, Gehrke SA, et al. G. Licochalcone A activates $\mathrm{Nrf} 2$ in vitro and contributes to licorice extract-induced lowered cutaneous oxidative stress in vivo. Exp Dermatol. 2015;24 (1):42-47. doi:10.1111/exd.2015.24.issue-1

20. Kornhauser A, Coelho SG, Hearing VJ. Applications of hydroxy acids: classification, mechanisms, and photoactivity. Clin Cosmet Investig Dermatol. 2010;24(3):135-142.

21. Sharad J. Glycolic acid peel therapy - a current review. Clin Cosmet Investig Dermatol. 2013;11(6):281-288. doi:10.2147/CCID.S34029

22. Arif T. Salicylic acid as a peeling agent: a comprehensive review. Clin Cosmet Investig Dermatol. 2015;26(8):455-461. doi:10.2147/CCID. S84765

23. Peirano RI, Hamann T, Düsing HJ, et al. Topically applied L-carnitine effectively reduces sebum secretion in human skin. J Cosmet Dermatol. 2012;11(1):30-36. doi:10.1111/j.1473-2165.2011.00597.x

\section{Publish your work in this journal}

Clinical, Cosmetic and Investigational Dermatology is an international, peer-reviewed, open access, online journal that focuses on the latest clinical and experimental research in all aspects of skin disease and cosmetic interventions. This journal is indexed on CAS
The manuscript management system is completely online and includes a very quick and fair peer-review system, which is all easy to use. Visit http://www.dovepress.com/testimonials.php to read real quotes from published authors. 